\section{Xantophyll-Zyklus in Chlamydomonas reinhardii}

\author{
Relef Kröning und Arminio Boschetti*
}

Abstract. During the xanthophyll cycle, which occurs upon illumination in thylakoid membranes of chloroplasts, violaxanthin is transformed via antheraxanthin into zeaxanthin. Not all of the violaxanthin, which seems to be present in a loosely and a more tightly bound form in pigment-protein complexes, participates in the cycle. Here we study the xanthophyll cycle of two pigment deficient mutants of Chlamydomonas reinhardii in order to get some information on the role and organization of the pigments in photosynthetic membranes. The mutant $p g-113$ has almost no chlorophyll $b$ and is reduced in neoxanthin, whereas mutant pg- $10 I$ contains no loroxanthin and normally only traces of lutein. After dark incubation, both mutants contain more violaxanthin per chlorophyll $a$ than the parent strain, from which the mutants have been derived. Upon illumination, in $p g-113$ violaxanthin is transformed into antheraxanthin and zeaxanthin, while in $p g-101$ taraxanthin and lutein might be produced. Since the amounts of transformed violaxanthin are higher in the mutants than in the parent strain, during illumination more NADPH (and ascorbate) is consumed in the mutants. We infer that in the mutants the pool of loosely bound violaxanthin as well as the ratio of loosely to tightly-bound pigment are higher than in the parent strain.

\section{Einleitung}

In grünen Pflanzen und Algen findet man Carotinoide in den Thylakoid-Membranen der Chloroplasten. Dort sind sie Bestandteile der Photosysteme 1 und II und der Lichtsammel-Chlorophyll-Protein-Komplexe. Carotinoid-defiziente Organismen, die nach Mutation auftreten, oder durch chemische Hemmung der Carotinoid-Biosynthese erzeugt werden, sind lichtempfindlich und sterben unter normalen Kulturbedingungen $\mathrm{ab}$ [1]. Obwohl Carotinoide in grünen Pflanzen und Algen offensichtlich lebensnotwendig sind, ist über ihre chemische Wirkungsweise wenig bekannt. Sie spielen einerseits eine Rolle bei der Lichtabsorption und Übertragung von Anregungsenergie auf die photosynthetischen Reaktionszentren und andererseits beim Schutz des Photosynthese-Apparates vor Photo-Oxydation [2]. Dabei scheinen sie die gelegentlich auftretenden Triplett-Zustände des Chlorophylls, welche Membranbestandteile schädigen können, abzubauen und die überaus toxischen Sauerstoff-Radikale, bzw. den Singlett-Sauerstoff abzufangen [3][4].

Möglicherweise ist an dieser Schutzfunktion der Carotinoide der XanthophyllZyklus massgeblich beteiligt (Schema) [5]. Bei diesem seit 1957 [6] bekannten Phänomen handelt es sich um die lichtabhängige, reversible Umwandlung dreier Xanthophylle, nämlich Violaxanthin, Antheraxanthin und Zeaxanthin. Bei starker Belichtung nimmt das Violaxanthin rasch ab und wird

* Korrespondenz: Prof. A. Boschetti

Institut für Biochemie

Universität Bern

Freiestr. 3, CH-3012 Bern auch rotes Licht, das nicht von den Carotinoiden sondern vom Chlorophyll absorbiert wird, aktiv ist [7].
Am Xanthophyll-Zyklus sind zwei auf gegenüberliegenden Seiten der ThylakoidMembran lokalisierte Enzyme beteiligt. Die Ascorbat-abhängige Deepoxydase mit einem Molekulargewicht von 54000 und einem pH-Optimum von 5,2 [7] liegt auf der Innenseite der Thylakoide. Das Enzym wird aktiviert, wenn der $\mathrm{pH}$ im Thylakoidlumen bei Belichtung absinkt. Die Oxygenase auf der Aussenseite der Thylakoide hat ein pHOptimum von 7,5 [7]. Sie katalysiert die Rückreaktion und erfordert $\mathrm{O}_{2}$ und NADPH. Obwohl das Enzym auch im Dunkeln arbeitet, kann die Umsetzung durch das im Licht gebildete NADPH stimuliert werden.

Die Lokalisierung der Deepoxydase und Oxygenase auf den entgegengesetzten Seiten der Thylakoid-Membran legt die Vermutung nahe, dass die am XanthophyllZyklus beteiligten Carotinoide nicht starr in Pigment-Protein-Komplexen fixiert sind, sondern sich quer zur Membran bewegen können. Vom Violaxanthin wurde gezeigt, dass es in mindestens zwei Formen auftritt, nämlich als fest und weniger fest in Chlorophyll-Protein-Komplexen gebundenes Pigment. Dabei ist das leicht gebundene Violaxanthin für den Zyklus verfügbar. $\mathrm{Ob}$ es in vivo in der Lipid-Membran als freies Molekül auftritt, lässt sich bis jetzt nicht sagen [8]. Da im Xanthophyll-Zyklus die Violaxanthinabnahme eine Reaktion erster Ordnung darstellt [9], scheint es nicht aus einem anderen Pool nachgeliefert zu werden, so dass ein Wechsel von Violaxanthin aus der fest gebundenen in die leicht gebundene Form höchstens sehr langsam ab-

Schema. Zwei Wege des Xanthophyll-Zyklus

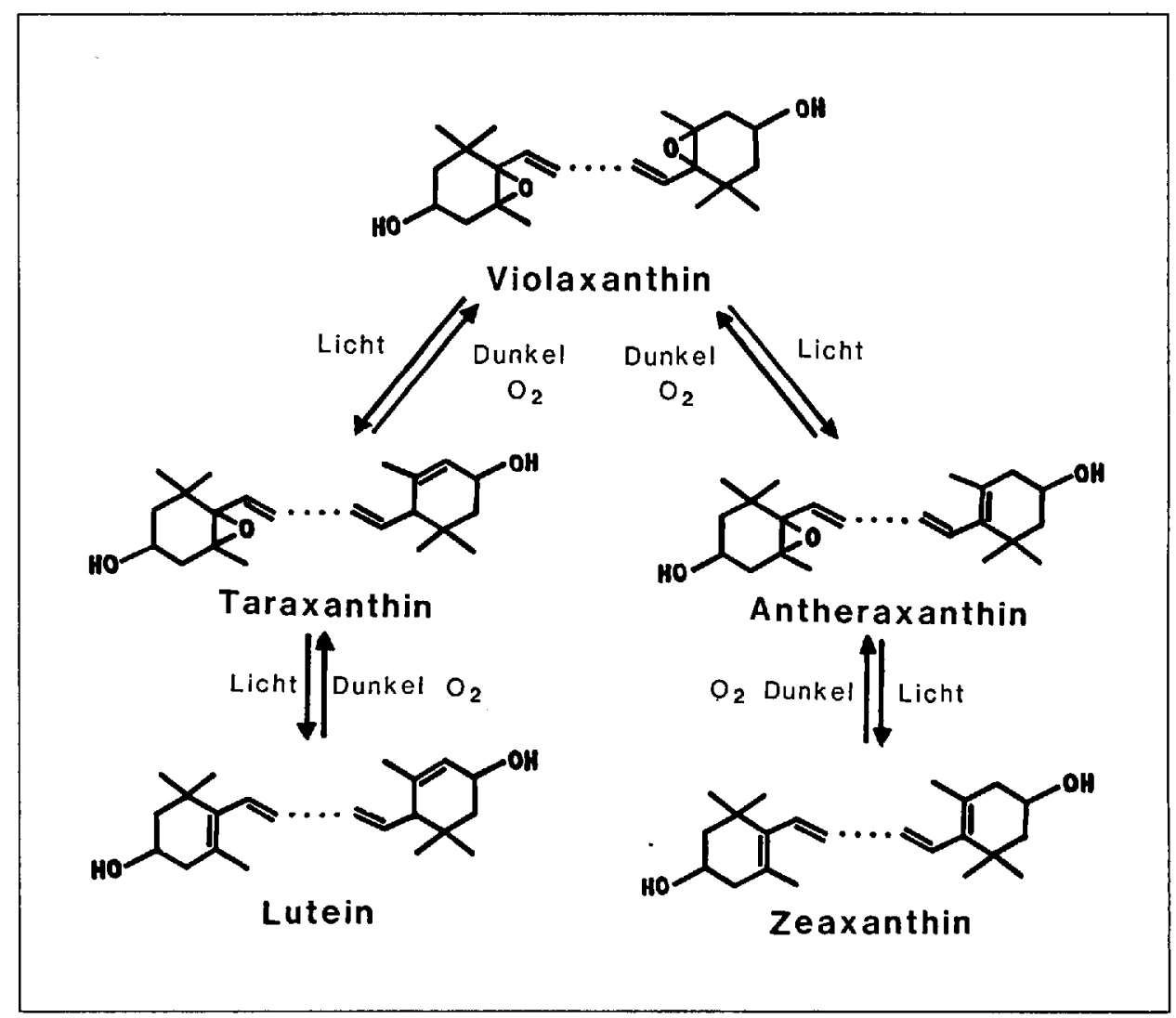




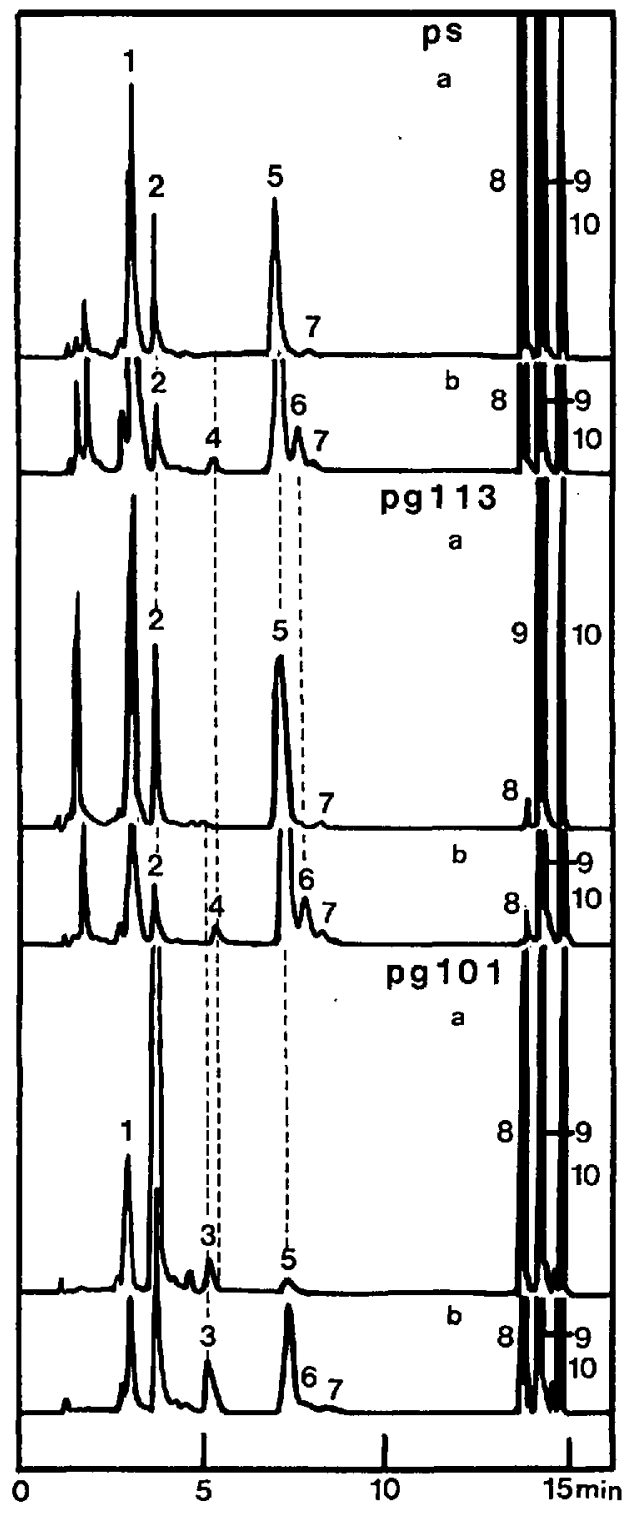

Fig 1.HPLC-Auftrennung der Pigmente aus dunkel-inkubierten (a) und belichteten (b) Kulturen von ps, pg-113 und pd-101. 1: Neoxanthin und Loroxanthin (überdeckt); 2: Violaxanthin; 3: Taraxanthin (Identifizierung auf Grund von Literaturvergleichen); 4: Antheraxanthin; 5: Lutein; 6: Zeaxanthin; 7: cis-Lutein(?); 8: Chlorophyll b; 9 : Chlorophyll $a$; 10: Carotin-Kohlenwasserstoffe.

läuft. So steht uns im Xanthophyll-Zyklus ein Instrument zur Erforschung der Pigmentorganisation innerhalb der ThylakoidMembran zur Verfügung. Bei Bestrahlung mit sättigenden Lichtintensitäten lässt sich annehmen, dass das Reaktionsgleichgewicht sehr weit auf der Seite des Zeaxanthins liegt, und dass die Menge des umgesetzten Violaxanthins näherungsweise der Menge des leicht gebundenen Pigmentes entspricht. In dem Masse, wie das Violaxanthin abnimmt sollte die Menge des Zeaxanthins ansteigen. Nach Erreichen des Gleichgewichtszustandes entspricht der Restgehalt an Violaxanthin weitgehend dem in den Pigment-Protein-Komplexen gebundenen, nicht dem Zyklus unterworfenen Pigment.
Auf diesen Aspekt hin untersuchten wir zwei Mutanten der Grünalge Chlamydomonas reinhardii deren Pigment-ProteinKomplexe gestört sind, und die vermutlich ein verändertes Verhältnis von fest- zu leichtgebundenem Pigment aufweisen. Die Mutante pg-113 enthält kein Chlorophyll $b$ und weniger Neoxanthin als der Ausgangsstamm, aus dem die Mutanten hergestellt worden sind [10]. Sie wächst auch etwas langsamer und ist lichtempfindlicher. Ihr Proteinmuster ist mit dem Ausgangsstamm vergleichbar. Dennoch fehlt ihr der prominente Lichtsammelkomplex LHC II, der normalerweise das meiste Chlorophyll $b$ und auch Violaxanthin enthält. Auch von der weniger gut charakterisierten zweiten Mutante pg-101 ist das Peptidmuster mit demjenigen des Ausgangsstammes vergleichbar. Die Mutante enthält aber kein Loroxanthin, unter normalen Kulturbedingungen fast kein Lutein und hat einen verminderten Chlorophyll- $b$-Gehalt [11]. Ein LHC II ist isolierbar, wenn auch in geringerer Menge als aus dem Ausgangsstamm.

\section{Experimentelles}

\section{Material und Methoden}

Die Kultivierung des verwendeten Ausgangssiammes Chlamydomonas reinhardii arg- $2 m t+(p s=$ parent strain) und der daraus hergestellten Mutanten $p g-1 / 3$ und $p g-101$ wurde in [12] beschrieben.

Unter Schütteln im Licht gewachsene 35-mlKulturen wurden bei einer Dichte von 5-6 $6 \times 10^{6}$ Zellen/ $\mathrm{ml}$ für $\mathrm{l} \mathrm{h}$ im Dunkeln inkubiert und dann bei $25^{\circ}$ im Schüttelwasserbad mit 65000 Lux (Philips Reflector $12535^{\circ}, 300 \mathrm{~W}$ ) belichtet. Nach verschiedenen Zeiten wurden die Kulturen durch Zugabe einer Spatelspitze $\mathrm{CaCO}_{3}$ und $140 \mathrm{ml}$ Aceton (Endkonz. 80\%) im Dunkeln während 5 min extrahiert. Nach Zentrifugation ( 5 $\min 2000 \times \mathrm{g}$ ) wurde der Oberstand mit $20 \% 4 \mathrm{M} \mathrm{NaCl}$

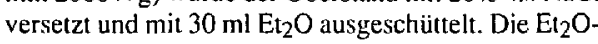
Phase wurde $2 \mathrm{mal}$ mit gleichem Volumen $0,4 \mathrm{M} \mathrm{NaCl}$ gewaschen und am $\mathrm{N}_{2}$-Strom eingeengt. Aufbewahrung unter $\mathrm{N}_{2}$ bei $-70^{\circ}$.

Zur HPLC-Auftrennung wurde der PigmentRückstand in 1-2 $\mathrm{ml} \mathrm{MeOH}$ aufgenommen und über eine Nucleosil 120-5-Cl8-Säule von $250 \times 4 \mathrm{~mm}$ (Machercy-Nagel, Oensingen) aufgetrennt. Laufmitte! 1: $\mathrm{MeCN} / \mathrm{MeOH} / \mathrm{H}_{2} \mathrm{O}$ 76:6:2; Laufmittel 2: THF/ MeOH 1:L; Programm: 9 min isokratisch Laufmittel 1 , Fluss $1,5 \mathrm{ml} / \mathrm{min} ; 4 \mathrm{~min}$ Gradient $0-100 \%$, Laufmittel 2, Fluss $2 \mathrm{ml} / \mathrm{min} ; 3 \mathrm{~min}$ isokratisch Laufmittel 2 ; Detektion: $440 \mathrm{~nm}$ (Fig. I). Die Pigment-Gehalte wurden berechnet unter Verwendung der molaren Extinktionskoeffizienten bei entsprechender Wellenlänge und Lsgm.-Zusammensetzung und auf die Menge Chlorophyll $a$ der Probe bezogen. Die Werte werden angegeben als pmol Pigment pro nmol Chlorophyll $a$.

\section{Resultate und Diskussion}

Die Kinetik der Xanthophyll-Zyklen beim Ausgangsstamm $p s$ und den Mutanten pg- 113 und $p g-101$ ist in Fig. 2 als zeitliche Veränderung der relativen Pigmentgehalte dargestellt.

ps: Bei dunkel inkubierten Zellen liegt der durchschnittliche, relative ViolaxanthinGehalt bei 273 (pmol Pigment/nmol Chlorophyll $a$ ). Er sinkt bei Belichtung auf 125 . Unter den gewählten Bedingungen werden also 148 Moleküle Violaxanthin pro 1000 Chlorophyll- $a$-Moleküle umgewandelt, was $54 \%$ der Anfangsmenge entspricht. Nach Belichtung steigt der relative Zeaxanthingehalt von $12 \mathrm{pmol} / \mathrm{nmol}$ Chlorophyll $a$ im Dunkeln auf 116 und derjenige von Antheraxanthin von 11 auf 54 an.

pg-113: Nach Dunkelinkubation liegt hier der relative Violaxanthin-Gehalt ca. 1,5mal höher als im Ausgangsstamm, nämlich bei $405 \mathrm{pmol} / \mathrm{nmol}$ Chlorophyll $a$. Nach Belichtung sinkt er auf einen mit dem Ausgangsstamm vergleichbaren Wert von 146. Dieses Violaxanthin scheint für den Zyklus nicht verfügbar zu sein und könnte ein Mass für das fest-gebundene Pigment darstellen. Die Menge des umgewandelten Pigmentes hingegen charakterisiert wahrscheinlich den Pool des leicht gebundenen Violaxanthins. Über die Versuchsdauer werden bei $p g-1 / 3$ somit pro 1000 Chlorophyll-a-Moleküle 260

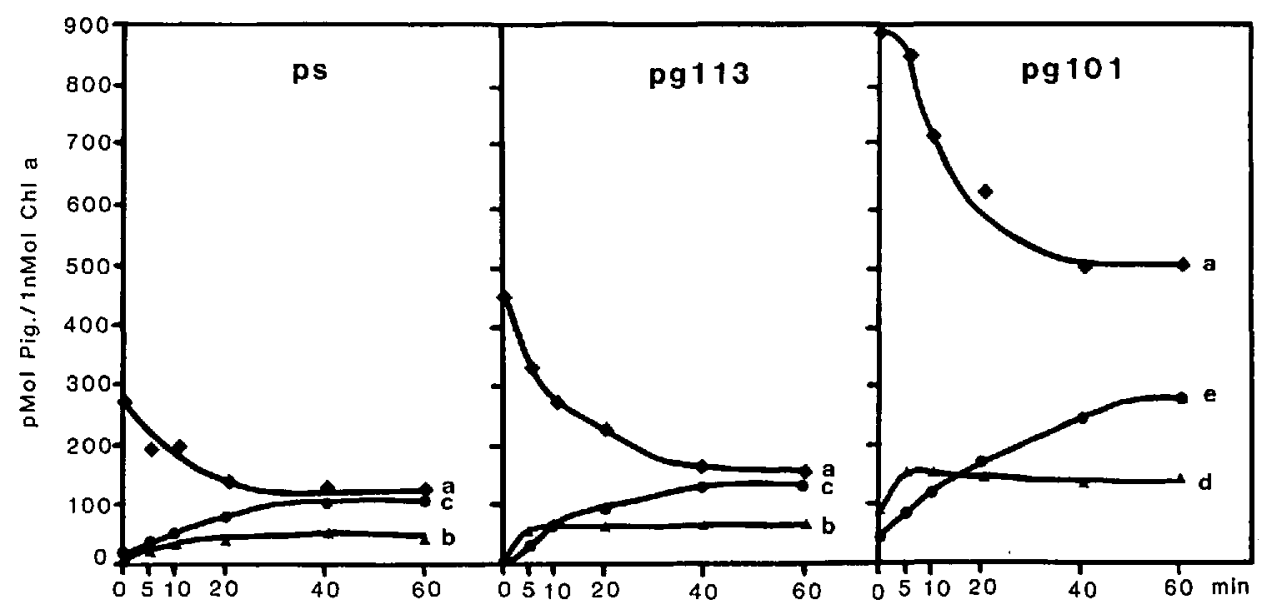

Fig. 2. Kinetischer Verlauf des Xanthophyll-Zyklus beim Ausgangsstamm ps sowie den Mutanten pg-113 und pg-101. Gemessen wurden die Gehaltsveränderungen in pmol Pigment pro nmol Chlorophyll $a$ bei Belichtung an Violaxanthin (a), Antheraxanthin bzw. Taraxanthin (?) bei pg-101 (b,d), Zeaxanthin bzw. Lutein (?) bei pg-101 $(c, e)$. Jede Kurve entspricht einer ausgewählten Einzelbestimmung. Im Text sind die Mittelwerte aus 2 bis 4 Experimenten angeführt. 
Violaxanthin-Moleküle umgesetzt, was 110 mehr sind als beim Ausgangsstamm und $64 \%$ der Anfangsmenge entspricht. Gleichzeitig steigt der relative AntheraxanthinGehalt von 0 auf 72 und der Zeaxanthingehalt von 1 auf 123 .

Der erhöhte Violaxanthin-Gehalt der gegenüber dem Ausgangsstamm ps etwas lichtempfindlicheren Mutante $p g-113$ könnte durch eine adaptive Verstärkung des Schutzmechanismus gegen Photo-Oxydation oder aber durch einen etwas geringeren Chlorophyll-Gehalt in der Mutante erklärt werden. Es ist auffallend, dass der Zeaxanthin-Spiegel nach Erreichen des Gleichgewichtes in beiden Stämmen fast gleich hoch ist. Entsprechend der Molsumme der drei Pigmente hat die Mutante dafür mehr Antheraxanthin in Gleichgewicht. Durch den grösseren Umsatz an Violaxanthin in $p g-113$ verbraucht die Mutante möglicherweise auch mehr Reduktionsmittel, Ascorbat und NADPH, als der Ausgangsstamm.

pg-101: Auffallend ist der extrem hohe relative Violaxanthin-Gehalt nach der Dunkel-Inkubation; er beträgt 870 pmol pro nmol Chlorophyll $a$, d.h. 3,2mal mehr als im Ausgangsstamm (Fig. 1 und 2). Nach Belichtung sinkt er nur auf 497. Dieser gegenüber den Stämmen ps und pg-113 3,8mal höhere Rest-Violaxanthin-Gehaltkönnte gemäss der einleitend geschilderten Hypothese auf einen grossen Pool festgebundenen Pigmentes hindeuten. Die umgewandelte, relative Menge an Violaxanthin beträgt 373 pmol pro nmol Chlorophyll $a$ also $43 \%$ der Ausgangsmenge. Das sind 1,4mal mehr als in $p g-113$ und $2,5 \mathrm{mal}$ als in $p s$. Parallel steigen die relativen Gehalte an Taraxanthin (?) von 81 auf 157, und von Lutein (?) von 40 auf 275. Auch hier vermuten wir also einen höheren Verbrauch an Reduktionsmitteln.

Die hier untersuchten Mutanten von Chlamydomonas reinhardii zeichnen sich primär durch ihr unterschiedliches Pigmentmuster und somit ihre gestörten Pigment-Protein-Komplexe aus. Obwohl sie vom selben Ausgangsstamm herstammen, zeigen sie zudem einen ganz anderen Verlauf des Xanthophyll-Zyklus. Möglicherweise erlaubt ihre weitere Untersuchung die Bedeutung der einzelnen Pigmente und des Xanthophyll-Zyklus in grünen Pflanzen etwas zu erhellen.

Wir danken Frau Dr. Siefermann-Harms, Karlsruhe für Beratung und Hilfestellung bei der Pigmentanalyse. Die Arbeit wurde teilweise durch den Schn'eiz. Nationalfonds unterstützt.
CHIMIA 45 (1991) Nr.1/2 (Jilluair/Fehruar)

Eingegangen am 11. Dezember 1990

[1] R.J.Cogdell, H.A. Frank, Binchim. Biophy's. Acta 1987, $895,63$.

[2] D. Siefermann-Harms, Physiol. Plant 1987, 69. 561 .

[3] N.I. Krinsky, Pure Appl. Chenı. 1979, 51, 649.

[4] B. Demmig-Adams, Nanuru'issenschafren 1989. $76,262$.

[5] D. Siefermann-Harms, in 'Lipids and lipid polymers in higher plants', Eds. M. Tevini und H.K. Lichtenthaler, Springer-Verlag, Berlin, 1977, S. 218.

[6] D.I. Sapozhnikov, T.A. Krasovskaya, A.N. Mayeskaya, Dokl. Akad. Nauk. USSR 1957,113. 465.

17] A. Hager, Ber. Disch. Bot. Ges. 1975, 88, 27.

[8] D. Siefermann-Harms, Photochem. Photohiol. $1984,40,507$.

[9] D. Siefermann, in 'Proc. 2nd Int. Congr. Photosynth.', Eds. G. Forti, M. Avron und A. Melandri, W. Junk Publ., 1972, S. 629

[10] H.P. Michel, M. Tellenbach, A. Boschenti, Biochim. Biophys. Acra 1983, 725, 417; M. Tellenbach, Dissertation, Universität Bern, 1984.

[11] A. S. Chunayev, N. G. Averina, A. Boschetti, E. M. Chekunova, N. V. Shalygo, in 'The topics of interaction of photosynthesis and respiration', Ed.V.L. Voznesensky, Tomsk University Press, 1988, S. 199; M. Tellenbach, Dissertation Bern, 1984.

[12] A. Boschetti, E. Sauton-Heiniger, J.C. Schaffner, W. Eichenberger, Physiol. Plant. 1978, 44. 134.
Chimia 45 (1991) 43-45

(C) Schweiz. Chemiker-Verband; ISSN 0009-4293

\section{Gas-phase Sequencing of Photoimmobilized Peptides}

\author{
Vibhuti Klingler-Dabral ${ }^{2}$ ), André Collioud ${ }^{1}$ ), Johann Schaller ${ }^{1}$ ), Franz Herbst ${ }^{2}$ ), \\ Wolf-Georg Forssmann ${ }^{2}$ ), and Hans Sigrist ${ }^{1}$ )*
}

Abstract. Aminopropylated glass-fiber discs were derivatized with the photosensitive reagents 3-(trifluoromethyl)-3-[m-(isothiocyano)phenyl]diazirine and $p$-azidophenyl isothiocyanate, respectively. Photolabel-derivatized solid supports were photoactivated in the presence of a hexapeptide. Gas-phase sequence analysis of washed fiber discs yielded end-to-end sequences with photoactivated disks only, indicating efficient photo-immobilization of the applied peptide. The quantity of amino acid released with each Edman-degradation cycle conferred with the established philicity of amino-acid side chains for photogenerated carbenes. The procedure provides a hitherto unexplored way to immobilize biomolecules (polypeptides) through statistically occurring carbene or nitrene insertions.

\section{Introduction}

Adsorption or covalent immobilization of polypeptides to solid supports are crucial steps in bio-analytical procedures including electrophoretic transfer of proteins from polyacrylamide gels to solid supports by electroblotting and immobilization of polypeptides for solid-phase and gas-phase sequence analysis. The efficiency of the methods mentioned depends on the protein-bin- ding capacity of the supporting material. To date, chemically inert supports are either pretreated or modified by various means to achieve improved polypeptide retention. For sequencing purposes, glass fibers are coated with polybrene, a quaternary ammonium polybase, or modified with positively of ionic forces to retain the adsorbed protein [1][2]. Covalently modified glass fibers, derived with bifunctional reagents, provide charged silanes. Both procedures make use means for group-specific protein binding [3][4]. Hydrophobic and electrostatic forces are involved in polypeptide retention on siliconized glass fiber [5] and polyvinylidenedifluoride (PVDF) membranes [6].

This study reports on the preparation and characterization of photoactivable supports, which carry monomolecular layers of carbene- or nitrene-generating photoreagents. Photo-activation of photolabel-derived supports in the presence of a hexapeptide leads to covalent polypeptide immobilization by carbene/nitrene insertion. Evidence is presented that photocoupled peptides can be sequenced by $E d m a n$-degradation-based gasphase microsequencing.

\section{Experimental}

Photoactivable Glass Fibers: Preparation and Loading

The bifunctional photoreagents 3-(trifluoromethyl)3-[m-(isothiocyano)phenyl]diazirine (TRIMID) and $p$ azidophenyl isothiocyanate (API) were synthesized as described in [7][8]. GF/C glass fiber sheets (Whatmam) were aminopropylated according to Aebersold et al. [2] yielding 14 nmol of $\mathrm{NH}$ groups per mg glass fiber. Dried aminopropylated glass-fiber sheets were cut into dises of $1.2 \mathrm{~cm}$ in diameter.

\footnotetext{
*Correspondence: PD Dr. Hans Sigrist

1) Institut für Biochemie

Universität Bern

Freiestrasse 3, CH-3012 Bern

2) Niedersächsisches Institut für Peptidforschung im Medical Park

Feodor-Lynen-Strasse 5, D-3000 Hannover 61
} 